\title{
Approximation of Curves by Line Segments
}

\section{By Henry Stone}

1. Introduction. Most methods of linear and nonlinear programming developed up to this time were designed to find the maximum or minimum value of a linear or nonlinear function inside a region bounded by hyperplanes. In applications of these methods it is often found that the formulation leads to constraints which are nonlinear. For example, in gasoline blending problems the relationship between lead concentration and octane number of a blend (the lead-susceptibility curve) has been found to be exponential in form. One method of dealing with such problems has been to approximate the given curves by a series of broken line segments [1]. The usual method of finding such an approximation has been, by visual examination of the graph, first to decide on the number of line segments, second, to select the intervals over which each line segment is to apply, and third, to draw in what appears to be good linear approximations to the curves over the selected intervals. Since the problem of obtaining a best fit of broken line segments to a curve does not seem to have been previously investigated, it is the purpose of this paper to formulate the problem, give a closed form solution when the given function is quadratic, show a general numerical method of solution, and apply this numerical procedure to the lead susceptibility curve.

In the case when the function is quadratic, an interesting and simple result was obtained. It was found that in fitting $N$ lines over some interval $(a, b)$, that the points at which one line segment was discontinued and the next line segment started were equally spaced over the interval. This result allowed the equations for the lines to be expressed in a very simple form.

\section{Formulation of the Problem. Given a known nonlinear relationship}

$$
y=f(x),
$$

$u_{0} \leqq x \leqq u_{N}$.

It is desired to obtain an approximation of the form

$$
y= \begin{cases}a_{1}+b_{1} x, & u_{0} \leqq x \leqq u_{1} \\ a_{2}+b_{2} x, & u_{1} \leqq x \leqq u_{2} \\ \cdots \cdots \cdots & \cdots \cdots \cdots \cdots \cdots \\ a_{N}+b_{N} x, & u_{N-1} \leqq x \leqq u_{N}\end{cases}
$$

which is best in the least squares sense. If the points $u_{1}, u_{2}, \cdots, u_{N}$ are specified in advance, the problem reduces to the simple case where the best fit over each interval is obtained separately by the usual least squares procedure. The results developed below will give the solution to this problem as a special case of the general solution. The more general case, treated in this paper, occurs when the end points of the line segments $u_{1}, u_{2}, \cdots, u_{N-1}$ are not specified in advance, and when only the number of line segments, $N$, is specified in addition to the relationship given by Equation (1). 
Following the least squares formulation, we wish to obtain the values of the variables which minimize

$$
\begin{aligned}
F\left(a_{1}, a_{2}, \cdots, a_{N}, b_{1}, b_{2}, \cdots, b_{N}, u_{1}, u_{2}, \cdots, u_{N-1}\right) \\
\quad=\sum_{j=1}^{N} \int_{u_{i-1}}^{u_{i}}\left(f(x)-a_{j}-b, x\right)^{2} d x .
\end{aligned}
$$

The method to be used is the usual normal equation technique of finding the point at which the partial derivatives of the function (2) are zero. This procedure leads to the following system of $3 N-1$ equations to be solved for the $3 N-1$ variables $a_{1}, \cdots, u_{N-1}$ :

$$
\begin{gathered}
\frac{\partial F}{\partial a_{p}}=0=-2 \int_{u_{p-1}}^{u_{p}} f(x) d x+2 a_{p}\left(u_{p}-u_{p-1}\right)+b_{p}\left(u_{p}^{2}-u_{p-1}^{2}\right) \\
p=1,2, \cdots, N \\
\frac{\partial F}{\partial b_{p}}=0=-2 \int_{u_{p-1}}^{u_{p}} x f(x) d x+a_{p}\left(u_{p}^{2}-u_{p-1}^{2}\right)+\frac{2}{3} b_{p}\left(u_{p}^{3}-u_{p-1}^{3}\right) \\
p=1,2, \cdots, N \\
\frac{\partial F}{\partial u_{p}}=0=2 f\left(u_{p}\right)\left(a_{p+1}-a_{p}\right)+2 u_{p} f\left(u_{p}\right)\left(b_{p+1}-b_{p}\right) \\
-2 u_{p}\left(a_{p+1} b_{p+1}-a_{p} b_{p}\right)-u_{p}^{2}\left(b_{p+1}^{2}-b_{p}{ }^{2}\right)-a_{p+1}^{2}+a_{p}^{2} \\
p=1,2, \cdots, N-1
\end{gathered}
$$

For the moment let us assume that the values of $u_{1}, \cdots, u_{N-1}$ are fixed. In this case the set of Equations (3a) and (3b) are seen to be nonhomogeneous and linear in terms of the $a$ 's and $b$ 's, and, further, each $a_{p}$ and $b_{p}$ pair may be obtained from the solutions of $N$ pairs of linear simultaneous equations. Carrying out this computation we obtain

$$
\begin{aligned}
& a_{p}=\frac{3}{\left(u_{p}-u_{p-1}\right)^{3}}\left\{\frac{4}{3}\left(u_{p}^{2}+u_{p} u_{p-1}+u_{p-1}^{2}\right) I\left(u_{p}, u_{p-1}\right)\right. \\
& \left.\quad-2\left(u_{p}+u_{p-1}\right) J\left(u_{p}, u_{p-1}\right)\right\} \\
& b_{p}=\frac{6}{\left(u_{p}-u_{p-1}\right)^{3}}\left\{2 J\left(u_{p}, u_{p-1}\right)-\left(u_{p}+u_{p-1}\right) I\left(u_{p}, u_{p-1}\right)\right\}
\end{aligned}
$$

where

$$
\begin{aligned}
& I\left(u_{p}, u_{p-1}\right)=\int_{u_{p-1}}^{u_{p}} f(x) d x, \\
& J\left(u_{p}, u_{p-1}\right)=\int_{u_{p-1}}^{u_{p}} x f(x) d x .
\end{aligned}
$$

Equations (4a) and (4b) represent the general solution to the problem when the points $u_{1}, \cdots, u_{N-1}$ have been selected in advance. When this is not the case then to proceed further we substitute the $a_{p}$ and $b_{p}$ from Equations (4) into Equations (3c), giving a system of $N-1$ nonlinear equations in $u_{1}, \cdots, u_{N-1}$ to be solved. In general no closed form solution for this set of equations can be found. In a later 
section of this report a numerical method of solution will be described which is practical for large-scale computer application. In the case when $f(x)$ is quadratic, some simplification of the problem occurs which allows a simple closed form solution to be obtained. This result is derived in the next section.

3. Solution for $f(x)$ Quadratic. In the case that $f(x)$ is quadratic, that is,

$$
f(x)=r x^{2}+s x+t,
$$

the function to be minimized is

$$
F=\sum_{j=1}^{N} \int_{u_{j-1}}^{u_{j}}\left(r x^{2}+s x+t-a_{j}-b_{j} x\right)^{2} d x .
$$

The integrand of this function can be simplified by consolidating the linear portion of $f(x)$ with the $a_{j}$ and $b_{j}$. So that, if we let .

$$
\begin{aligned}
& \alpha_{j}=\left(a_{j}-t\right) / r \\
& \beta_{j}=\left(b_{j}-s\right) / r,
\end{aligned}
$$

Equation (6) becomes

$$
F=r^{2} \sum_{j=1}^{N} \int_{u_{i-1}}^{u_{i}}\left(x^{2}-\alpha_{j}-\beta_{j} x\right)^{2} d x
$$

and it is only necessary to find the minimum of Equation (8) with respect to the $\alpha_{j}, \beta_{j}$ and $u_{j}$. The constant multiplier, $r^{2}$, in (8) drops out in the derivation of the normal equations.

Following the procedure described in the last section with now $f(x)=x^{2}$, the $\alpha_{p}$ and $\beta_{p}$ are obtained from Ecyuations (4) and have the following simple form:

$$
\begin{array}{ll}
\alpha_{p}=-\frac{1}{6}\left[\left(u_{p}+u_{p-1}\right)^{2}+2 u_{p} u_{p-1}\right] & \\
\beta_{p}=u_{p}+u_{p-1}, & p=1,2, \cdots, N .
\end{array}
$$

Before proceeding to the solution of the general problem, let us first examine the case of $N=2$. In this case Equations ( $3 \mathrm{c}$ ) reduce to a single equation in $u$. Substituting the results of Equations (9) into (3c) we obtain after some straightforward but tedious algebra the following equation in $u_{1}$.

$$
\text { (10) } 4\left(u_{2}-u_{0}\right) u_{1}^{3}-6\left(u_{2}^{2}-u_{0}^{2}\right) u_{1}^{2}+4\left(u_{2}^{3}-u_{0}^{3}\right) u_{1}-\left(u_{2}^{4}-u_{0}^{4}\right)=0
$$

which has for its only real root

$$
u_{1}=\frac{1}{2}\left(u_{2}+u_{0}\right) \text {. }
$$

With $u_{1}$ given by (11) and $\alpha_{1}, \beta_{1}, \alpha_{2}, \beta_{2}$ by (9), it is easy to rerify that

$$
\alpha_{1}+\beta_{1} u_{1}=\alpha_{2}+\beta_{2} u_{1}
$$

and in terms of the original variables

$$
a_{1}+b_{1} u_{1}=a_{2}+b_{2} u_{1} ;
$$

that is, the lines of best fit intersect at their common end point.

To proceed now with the case of fitting.$V$ lines to the quadratic, we now make 
the additional continuity assumption, verified in the case $N=2$, that each pair of adjacent lines intersect at their end points, $u_{1}, u_{2}, \cdots u_{N-1}$, so that instead of the system of nonlinear equations (3c) to solve, we have instead the system

$$
\alpha_{p}+\beta_{p} u_{p}=\alpha_{p+1}+\beta_{p+1} u_{p}, \quad p=1,2, \cdots, N-1,
$$

and substituting for $\alpha_{p}$ and $\beta_{p}$ from (9) we have

$$
2 u_{p} u_{p-1}-u_{p-1}^{2}=2 u_{p} u_{p+1}-u_{p+1}^{2}, \quad p=1,2, \cdots, N-1
$$

which further simplifies to

$$
u_{p-1}-2 u_{p}+u_{p+1}=0, \quad p=1,2, \cdots, N-1 .
$$

Equation (16) may be regarded as a system of linear homogeneous difference equations, with two known boundary points, $u_{0}$ and $u_{N}$. The general solution is of the form

$$
u_{p}=k_{1}+k_{2} p,
$$

and evaluating the constants, $k_{1}$ and $k_{2}$, from the conditions at $p=0$ and $p=N$, we have finally

$$
u_{p}=u_{0}+\frac{p}{N}\left(u_{N}-u_{0}\right), \quad p=1,2, \cdots, N-1
$$

as the values for the common end points. This equation gives the interesting result that for the continuous case, the end points are spaced out at equal intervals over the whole interval $\left(u_{0}, u_{N}\right)$. In applications of the method it has been found most convenient first to determine the $u$ 's from (18), next calculate the $\alpha$ 's and $\beta$ 's from (9) and finally the $a$ 's and $b$ 's from

$$
\begin{aligned}
& a_{p}=r \alpha_{p}+t \\
& b_{p}=r \beta_{p}+s, \quad \quad p=1,2, \cdots, N
\end{aligned}
$$

where $r, s, t$ are the coefficients of the original quadratic (j).

4. A Numerical Method of Solution. The basic problem is to find a solution of the normal equations (3); that is, to find the $a$ 's, $b$ 's and $u$ 's satisfying

$$
\begin{array}{lr}
\frac{\partial F}{\partial a_{p}}=0, & p=1,2, \cdots, N \\
\frac{\partial F}{\partial b_{p}}=0, & p=1,2, \cdots, N \\
\frac{\partial F}{\partial u_{p}}=0, & p=1,2, \cdots, N-1 .
\end{array}
$$

It has been shown that these equations are usually so complicated that the solution cannot be obtained directly. A general method in such cases is to assume a trial solution and derive linear equations for small additive corrections. The solution of this set of linear equations leads to a new set of trial values and the process can be repeated until the corrections become negligible. 
Description of the method becomes much simpler if we adopt a new notation. To this end let

$$
\begin{aligned}
& \theta_{1}=a_{1}, \quad \theta_{2}=a_{2}, \quad \cdots, \quad \theta_{N}=a_{N}, \\
& \theta_{N+1}=b_{1}, \quad \theta_{N+2}=b_{2}, \quad \cdots, \quad \theta_{2 N}=b_{N}, \\
& \theta_{2 N+1}=u_{1}, \quad \theta_{2 N+2}=u_{2}, \quad \cdots, \quad \theta_{3 N-1}=u_{N-1} \text {. }
\end{aligned}
$$

The system of equations to be solved is then

$$
\frac{\partial F}{\partial \theta_{p}}=0, \quad \quad p=1,2, \cdots, 3 N-1
$$

Let the trial values be denoted by $\theta_{1}^{0}, \cdots, \theta_{3 N-1}^{0}$; expanding $\left(3^{\prime}\right)$ in Taylor's series about the point $\theta^{\theta}$, and dropping higher-order terms, we have

$$
\frac{\partial F}{\partial \theta_{p}}=0 \simeq \frac{\partial F}{\partial \theta_{p}{ }^{0}}+\sum_{j=1}^{3 N-1}\left(\theta_{j}-\theta_{j}{ }^{0}\right) \frac{\partial^{2} F}{\partial \theta_{p}{ }^{0} \partial \theta_{j}{ }^{0}}, \quad p=1, \cdots, 3 N-1
$$

where the notation $\left(\partial F / \partial \theta_{p}{ }^{0}\right)$, etc. means that the indicated derivative is to be evaluated at the point $\theta_{0}$. Let $\Delta \Theta=\Theta-\Theta^{0}$ be the vector with components, $\theta_{1}-\theta_{1}{ }^{0}, \cdots, \theta_{3 N-1}-\theta_{3 N-1}^{0}$, let $G$ be the vector with components, $\left(\partial F / \partial \theta_{1}{ }^{0}\right)$, $\cdots,\left(\partial F / \partial \theta_{3 N-1}^{0}\right)$, and let $H$ be the $(3 N-1)$ by $(3 N-1)$ matrix whose $(i, j)$ component is $\left(\partial^{2} F / \partial \theta_{i}{ }^{0} \partial \theta_{j}{ }^{0}\right)$. Then in matrix notation the right-hand side of Equation (20) becomes

$$
G+\Delta \Theta \cdot H=0
$$

and if $H$ is non-singular, then $\Theta^{\prime}$, the second approximation to the solution of (20), is given by

$$
\Theta^{1}=\Theta^{0}-H^{-1} G
$$

A FORTRAN computer program for the IBM 704 has been written and tested which will solve this problem for any function, $f(x)$, which is differentiable. A FORTRAN subroutine must be supplied for each $f(x)$, which will compute $f(k)$, $I\left(k_{1}, k_{2}\right), J\left(k_{1}, k_{2}\right)$ and $(\partial f(k) / \partial k)$. Further details can be supplied by the author.

5. Application to Lead Susceptibility Curves. As described in the Introduction, part of the purpose of the research described in this paper was to find the best method of linearizing the lead susceptibility curve. It has been found experimentally that for every blend examined, the relationship between octane number of the blend and quantity of tetraethyl lead added is adequately given by

$$
O \cdot N \cdot=k_{1}+k_{2} e^{-c x}=f(x),
$$$$
0 \leqq x \leqq 3.0
$$

where $O \cdot N$. is the octane number of the blend, $x$ is the quantity of lead in c.c., and $k_{1}, k_{2}, c$ are known constants representative of the blend. It has been indicated above that this problem may be treated as a single-parameter problem in $c$ alone, since $k_{1}$ and $k_{2}$ may be absorbed into the fitted lines. Thus, instead of having the integrand of Equation (2) represented by terms of the form

$$
\left(k_{1}+k_{2} e^{-c x}-a_{j}-b_{j} x\right)^{2}
$$


let us set

$$
\begin{aligned}
& \alpha_{j}=\left(a_{j}-k_{1}\right) / k_{2} \\
& \beta_{j}=b_{j} / k_{2},
\end{aligned}
$$

and the integrand becomes proportional to

$$
\left(e^{-c x}-\alpha_{j}-\beta_{j} x\right)^{2} .
$$

The method and computer program described in the last section were used to obtain values of the line and end point parameters for fitting two, three and four line segments to the exponential function, $\exp (-c x)$. The accompanying Tables, 1 through 3 , give the normalized line slopes, $\beta$, the normalized intercepts, $\alpha$, and the values of the end points, $u$, for the number of lines, $N$, equal to $2,3,4$, as a function

TABLE 1

Line Parameters, $N=2$

\begin{tabular}{c|c|c|c|c|c}
\hline \multirow{2}{*}{$c$} & \multicolumn{2}{|c|}{ Intercepts } & \multicolumn{2}{c}{ Slopes } \\
\cline { 2 - 5 } & $\alpha_{1}$ & $\alpha_{2}$ & $\beta_{1}$ & $\beta_{2}$ & \\
\cline { 2 - 5 } & 0.998 & 0.980 & -0.093 & -0.080 & 1.385 \\
0.1 & 0.994 & 0.931 & -0.174 & -0.129 & 1.400 \\
0.3 & 0.988 & 0.867 & -0.246 & -0.157 & 1.360 \\
0.4 & 0.981 & 0.798 & -0.309 & -0.170 & 1.316 \\
0.5 & 0.973 & 0.728 & -0.367 & -0.175 & 1.276 \\
0.6 & 0.966 & 0.661 & -0.420 & -0.173 & 1.235 \\
0.7 & 0.958 & 0.598 & -0.469 & -0.168 & 1.196 \\
0.8 & 0.950 & 0.540 & -0.515 & -0.160 & 1.155 \\
0.9 & 0.943 & 0.488 & -0.558 & -0.152 & 1.116 \\
1.0 & 0.935 & 0.442 & -0.600 & -0.142 & 1.080 \\
1.2 & 0.923 & 0.363 & -0.680 & -0.124 & 1.008 \\
1.5 & 0.906 & 0.275 & -0.793 & -0.101 & 0.912 \\
\hline
\end{tabular}

TABLE 2

Line Parameters, $N=3$

\begin{tabular}{c|c|c|c|c|c|c|c|c}
\hline & \multicolumn{3}{|c|}{ Intercepts } & \multicolumn{4}{c|}{ Slopes } & \multicolumn{2}{c}{ End Points } \\
\cline { 2 - 9 } & $\alpha_{1}$ & $\alpha_{2}$ & $\alpha_{3}$ & $\beta_{1}$ & $\beta_{2}$ & $\beta_{2}$ & $u_{1}$ & $u=$ \\
\hline 0.1 & 0.999 & 0.993 & 0.976 & -0.096 & -0.088 & -0.079 & 0.785 & 1.783 \\
0.2 & 0.998 & 0.975 & 0.919 & -0.186 & -0.156 & -0.125 & 0.782 & 1.775 \\
0.3 & 0.995 & 0.943 & 0.840 & -0.266 & -0.202 & -0.146 & 0.835 & 1.820 \\
0.4 & 0.992 & 0.907 & 0.756 & -0.340 & -0.237 & -0.154 & 0.830 & 1.810 \\
0.5 & 0.988 & 0.870 & 0.673 & -0.411 & -0.265 & -0.153 & 0.801 & 1.773 \\
0.6 & 0.985 & 0.835 & 0.595 & -0.478 & -0.285 & -0.147 & 0.774 & 1.738 \\
0.7 & 0.981 & 0.798 & 0.523 & -0.541 & -0.299 & -0.137 & 0.756 & 1.708 \\
0.8 & 0.978 & 0.767 & 0.460 & -0.604 & -0.312 & -0.127 & 0.724 & 1.663 \\
0.9 & 0.974 & 0.737 & 0.404 & -0.665 & -0.323 & -0.117 & 0.694 & 1.618 \\
1.0 & 0.971 & 0.711 & 0.355 & -0.725 & -0.333 & -0.107 & 0.666 & 1.573 \\
1.2 & 0.966 & 0.664 & 0.275 & -0.841 & -0.349 & -0.088 & 0.615 & 1.487 \\
1.5 & 0.959 & 0.608 & 0.192 & -1.012 & -0.371 & -0.066 & 0.548 & 1.365 \\
\hline
\end{tabular}


TABLE 3

Line Parameters, $N=4$

\begin{tabular}{|c|c|c|c|c|c|c|c|c|c|c|c|}
\hline \multirow{2}{*}{$c$} & \multicolumn{4}{|c|}{ Intercepts } & \multicolumn{4}{|c|}{ Slopes } & \multicolumn{3}{|c|}{ End Points } \\
\hline & $\alpha_{1}$ & $\alpha_{2}$ & $\alpha_{\mathbf{z}}$ & $\alpha_{4}$ & $\beta_{1}$ & $\beta_{2}$ & $\boldsymbol{B}_{\mathbf{z}}$ & $\boldsymbol{B}_{\mathbf{t}}$ & $u_{1}$ & $u_{2}$ & $u_{\mathbf{z}}$ \\
\hline 0.1 & 1.000 & .996 & .988 & .973 & -0.097 & -0.091 & -0.085 & $-0.07 \varepsilon$ & .57 & 1.268 & 2.07 \\
\hline 0.2 & 0.99 & 98 & 9 & (3) & -0.188 & -0.166 & -0.143 & -0.12 & 0.60 & .29 & 2.096 \\
\hline 0.3 & 0.997 & 0.968 & 91 & .824 & 0.274 & -0.226 & -0.181 & -0.140 & 0.607 & 1.293 & 2.087 \\
\hline .4 & 0.996 & 0.948 & $.8 \% 9$ & 0.734 & 0.356 & -0.276 & -0.206 & -0.146 & 0.595 & 1.275 & 2.068 \\
\hline.$j$ & 0.999 & $0 . !$ & . & & 34 & & -0.221 & 43 & 77 & 1.24 & 2.040 \\
\hline 0.6 & 0.992 & 0.90 & 0.75 & $0.5)(62$ & -0.508 & -0.352 & -0.228 & -0.134 & 0.563 & 1.222 & 2.01 \\
\hline 0.7 & 0.989 & 0.880 & 0.700 & 0.486 & -0.580 & -0.381 & -0.231 & -0.124 & 0.549 & $1.199 \mid$ & 1.99 \\
\hline 8 & 0.987 & (0.85! & $0 .(5 ; 54$ & 0.420 & -0.6 .50 & -0.409 & -0.232 & -0.112 & 0.530 & $1.165 \mid$ & 1.95 \\
\hline 9 & $0.98 i$ & 0.84 & . 61 & $0.36 ; 3$ & -0.720 & -0.435 & -0.233 & -0.101 & $|0.507|$ & 1.124 & 1.910 \\
\hline & .98 & 82 & 57 & 313 & -0.789 & -0.460 & -0.232 & -0.091 & $0.485 \mid$ & 1.08 & 1.86 \\
\hline & .98 & $7 !$ & 51 & 234 & -0.925 & - & -0.230 & -0 & 0.4 & .008 & .778 \\
\hline & & & & & 126 & 574 & .227 & & & 0.907 & \\
\hline
\end{tabular}

TABLE 4

Maximum Fitting Errors

\begin{tabular}{c|c|c|c}
\hline & \multicolumn{3}{|c}{$\max \left(e^{-c x}-\alpha_{j}-\beta_{j} x\right)$} \\
\cline { 2 - 3 } & $N=2$ & $N=3$ & $N=4$ \\
\hline 0.1 & 0.0016 & 0.0010 & 0.00055 \\
0.2 & 0.0059 & 0.0032 & 0.0016 \\
0.3 & 0.0119 & 0.0050 & 0.0028 \\
0.4 & 0.0189 & 0.0080 & 0.0043 \\
0.5 & 0.0265 & 0.0114 & 0.0062 \\
0.6 & 0.0344 & 0.0150 & 0.0083 \\
0.7 & 0.0423 & 0.0190 & 0.0106 \\
0.8 & 0.0500 & 0.0223 & 0.0127 \\
0.9 & 0.0574 & 0.0255 & 0.0145 \\
1.0 & 0.0645 & 0.0286 & 0.0162 \\
1.2 & 0.0774 & 0.0341 & 0.0193 \\
1.5 & 0.0936 & 0.0410 & 0.0231 \\
\hline
\end{tabular}

of the exponential parameter, $c$. The actual intercepts and slopes, $a$ and $b$, for any particular problem are obtained from

$$
\begin{aligned}
& a_{j}=k_{1}+k_{2} \alpha_{j} \\
& b_{j}=k_{2} \beta_{j} .
\end{aligned}
$$

It has been found preferable in practice, for reasons of accuracy, to determine the common end points of the lines from the relation

$$
a_{j}+b_{j} u_{j}=a_{j+1}+b_{j+1} u_{j}
$$

giving

$$
u_{j}=\frac{a_{j}-a_{j+1}}{b_{j+1}-b_{j}} .
$$

In order to provide the user of this method with a guide to the number of line segments that should be used for any particular application, an error analysis was 
carried out. The maximum difference between the exponential function, $e^{-e x}$, and the lines $a_{j}-b_{j} x$ were computed for the two, three, and four line cases. The results are shown in Table 4 where

$$
\max \left(e^{-c x}-\alpha_{j}-\beta_{j} x\right), \quad 0 \leqq x \leqq 3.0
$$

is given as a function of $c$ for these cases. To transform the error given in the normalized form to error in terms of octane number for any particular case, the transformation

$$
\text { Error }(O \cdot N \cdot)=k_{2} \cdot \text { Error (Table) }
$$

is used where $k_{2}$ is the constant shown in Equation (23); that is, in the equation

$$
O \cdot N \cdot=k_{1}+k_{2} e^{-c x} \text {. }
$$

The tables given for the lead susceptibility curves can be used for curves of a more general character, namely those of the form

$$
g(z)=r_{1}+r_{2} z+r_{3} e^{-r_{4} z}, \quad u_{0} \leqq z \leqq w_{s} .
$$

In this case the least squares function is

$$
F=\sum_{j=1}^{N} \int_{w_{j-1}}^{w_{i}}\left(r_{1}+r_{2} z+r_{3} e^{-r_{4} z}-a_{j}-b_{j} z\right)^{2} d z .
$$

Let a new variable $x$ be defined by

$$
x=3 \cdot \frac{z-w_{0}}{w_{N}-w_{0}}, \quad d x=\frac{3 d z}{w_{N}-w_{0}} .
$$

The range of $x$ is $0 \leqq x \leqq 3.0$, and $F$ becomes

$$
F \propto \sum_{j=1}^{N} \int_{u_{j}-1}^{u_{j}}\left(e^{-c x}-\alpha_{j}-\beta_{j} x\right) d x
$$

which is identical in form, except for a proportionality constant, to the previous case. The transformation equations are

$$
\begin{aligned}
\alpha_{j} & =\frac{1}{r_{3}}\left[a_{j}-r_{1}+\left(b_{j}-r_{2}\right) w_{0}\right] \\
\beta_{j} & =\left(\frac{w_{N}-w_{0}}{3}\right)\left(b_{j}-r_{2}\right) \\
u_{j} & =3\left(\frac{w_{j}-w_{0}}{w_{N}-w_{0}}\right) .
\end{aligned}
$$

The inverse transformation equations to the original units are

$$
\begin{aligned}
b_{j} & =r_{2}+\frac{3 \beta_{j}}{w_{N}-w_{0}} \\
a_{j} & =r_{1}+r_{3} \alpha_{j}-\left(b_{j}-r_{2}\right) w_{0} \\
w_{j} & =w_{0}+\left(\frac{w_{N}-w_{0}}{3}\right) u_{j} .
\end{aligned}
$$

Shell Development Company

Emeryville, California

1. W. W. Garvin, H. W. Crandall, J. B. John, R. A. Spellman, "Applications of linear programming in the oil industry,"'Management $S c i .3,1957$, p. $40 \vec{r}$. 